\title{
Nanostructured ZnO for biosensing applications
}

\author{
XU ChunXiang $^{1 *}$, YANG Chi $^{1}$, GU BaoXiang ${ }^{2}$ \& FANG ShengJiang ${ }^{1}$ \\ ${ }^{1}$ State Key Laboratory of Bioelectronics, School of Electronic Science and Engineering, Southeast University, Nanjing 210096, China; \\ ${ }^{2}$ Department of Resources and Environment Engineering, Henan Institute of Engineering, Zhengzhou 450000, China
}

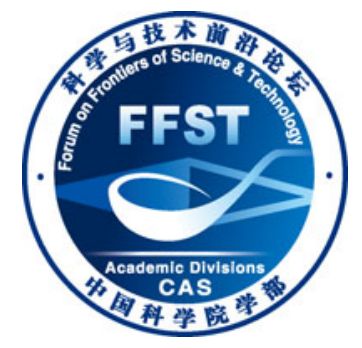

Received July 5, 2012; accepted September 3, 2012; published online May 6, 2013

\begin{abstract}
Based on the unique properties, nanostructured $\mathrm{ZnO}$ could provide a stable immobilization for biomolecules retaining their biological activity. It has been recently developed as a nice candidate for the construction of biosensors with enhanced analytical performance. In this paper, we reviewed the progress in adapting nanostructured $\mathrm{ZnO}$ for several predominantly in biosensing applications based on enzymic reaction, immunoreaction, and molecular compitation. We also described several important considerations when working with nanostructured $\mathrm{ZnO}$ mainly centered on the fabrications of $\mathrm{ZnO}$ and appropriate strategies for biosensor construction (e.g. modified electrodes and multilayered immobilization).
\end{abstract}

ZnO, biosensor, Zn alloy

Citation: $\quad$ Xu C X, Yang C, Gu B X, et al. Nanostructured ZnO for biosensing applications. Chin Sci Bull, 2013, 58: 2563-2566, doi: 10.1007/s11434-013-5714-5

Semiconductor biosensor is based on combining the properties of biologically active materials with those of inorganic crystalline materials that translate that translate the biological properties into electronic signals. These signals can then be processed to identify the properties of biological elements. [1]. Zinc oxide has attracted much attention particularly due to its widely applications in optics, optoelectronics, sensors, and actuators [2,3]. The biocompatibility, low toxicity, high electron mobility and easy fabrication of $\mathrm{ZnO}$ are favorable for biosensing [4-7]. Moreover, it has a high isoelectric point (IEP) of about 9.5, which makes it suitable for adsorption of proteins with low IEPs via electrostatic interactions [7]. Meanwhile, $\mathrm{ZnO}$ has probably the richest variety of different nanostructures which is benefit for biosensor design. So far, $\mathrm{ZnO}$ combs, nanotubes and nanorods have been employed to enzyme biosensor, immunosensor and other type sensor [8-12]. Especially, $\mathrm{ZnO}$ quantum dots (QDs) which have similar size as the biomolecules provide a good interactive environment for the biomarker $[13,14]$. The scope of this article is focused on our recent research

*Corresponding author (email: xcxseu@ seu.edu.cn) progress on interaction/integration of $\mathrm{ZnO}$ QDs and $\mathrm{ZnO}$ one-dimensional (1-D) nanostructure with biomolecules for sensor applications.

\section{$1 \mathrm{ZnO}$ quantum dots biosensor}

$\mathrm{ZnO}$ QDs as high aspect nanoparticles with high IEP have been promised as probes for ultrasensitive detection of cancer biomarkers [13,14]. Their fluorescent properties have enabled $\mathrm{ZnO}$ QDs to be used as labels for in vitro assays to quantify biomarkers, for example, the conjugates of $\mathrm{ZnO}$ QDs with antibodies, aptamers, oligonucleotides, or peptides can act as the target cancer markers. ZnO QDs have also been exploited for electrochemical detection on the basis of their elemental compositions besides optical approach. Owing to the amplification effect originating from dissolving $\mathrm{ZnO}$ QDs, and the highly sensitive nature of electrochemical stripping detection, the sensitivity of electrochemical detection methods for cancer biomarkers is very high.

Carbohydrate antigen 19-9 (CA 19-9) is a preferred label for pancreatic tumor. It is a malignant tumor and difficult to 
be early diagnosed in current clinical medicine [15]. In our previous work [16], using $\mathrm{ZnO}$ QDs as electrochemical and fluorescent labels, a sandwich-type sensitive immunoassay was developed based on immunoreactions between the antibody and antigen to detect the antigen CA19-9. Stripping voltammetry and photoluminescence were conducted to carry out an efficient immunoassay based on the electrochemical and optical properties of $\mathrm{ZnO}$ QDs. The immunosensor showed high sensitivity, stability, and reproducibility. The spectral measurement presented a linear range from 1 to 180 $\mathrm{U} \mathrm{mL}^{-1}$ with a detection limit of $0.25 \mathrm{U} \mathrm{mL}^{-1}$. The detection limit was further reduced to $0.04 \mathrm{U} \mathrm{mL}^{-1}$ and the linear range was extended from 0.1 to $180 \mathrm{U} \mathrm{mL}^{-1}$ by square wave voltammetry (SWV) analysis, as shown in Figure 1. The specificity experiments were further verified by competition experiments to evaluate the association of CA 19-9 antigen with QD labeled CA 19-9 antibody probe in the presence of increasing concentrations of nonspecific latent membrane protein competitors. The results show that $\mathrm{ZnO}$ QD-based immunosensor has a good specificity for CA 19-9 detection.

$\mathrm{ZnO}$ QDs were also employed in the lectin biosensor. As we know, as compared to the immunosensor, the lectin biosensor could obviate time-consuming, high cost and denaturation in existing methods. More important, some lectins were found to be as specific as antibody binds to a specific antigen and applied in the biosensor [17]. We report a direct electrochemical biosensor for the detection of allergy chicken ovomucoid (CHOM) based on bioconjugates of $\mathrm{ZnO}$ QDs binding CHOM [18]. The CHOM bioconjugates is formed through electrostatic interaction between positively charged QDs and negatively charged protein. Concanavalin A (Con A) was employed as a recognition element for the $\mathrm{CHOM}$ bioconjugates. The extent detection was accomplished by electrochemical SWV analysis of cadmium released by acid from the captured quantum dots. The biosensor shows concentration-dependent SWV development by capturing CHOMfunctiona-lized $\mathrm{ZnO}$ QDs on Con A-coated chips and has a detection limit of $1 \mathrm{ng} \mathrm{mL} \mathrm{m}^{-1}$. As an important criterion for any analytical tool, the biosensor shows acceptable specificity towards CHOM.

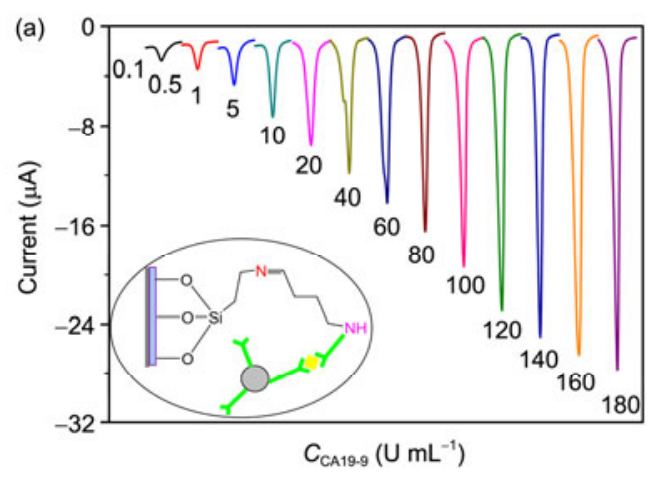

Although the above proposed method makes the analyte was detected directly through electrochemical of the $\mathrm{ZnO}$ analyte bioconjugates captured on Con A substrate, the analytes have to be labeled each time. To avoid this problem, a substitution method was developed using carcinoembryonic antigen (CEA) as a model. The basic principle is that the $\mathrm{ZnO}-\mathrm{CEA}$ bioconjugates could be replaced by the analyte CEA captured on the Con A substrate in advance because the former combined more weakly than the later combined with Con A. The analyte CEA is monitored by electrochemical of the undisplacement $\mathrm{ZnO}$-CEA bioconjugates on the Con A substrate [19]. The assay was based on the competition between a quantum dots labeled CEA and analyte CEA using concanavalin A as the recognition element [20]. The extent of completion was monitored by the square wave stripping voltammetry. The results show that the sensor demonstrated an acceptable precision, reproducibility and storage stability.

The competition method is expected to develop an approach for multi-analytic determination simultaneously by tagging each biomolecule with different QDs. As an example, the pharmacology (QP) of cetuximab (C225), a monoclonal antibody directed against colorectal cancer, and the biomarker of the cancer, CEA were designed to analyze quantitatively. In order to find the optimal dose for each individual patient, we usually need to get the dynamic changes of CEA (biomarker of the colorectal) and C225 in advance. Thus, simultaneous detection of CEA and C225 is the first step toward an assay for QP of C225. We developed a $\mathrm{ZnO}$ and $\mathrm{CdSe}$ QDs-based multi-analyte biosensor for simultaneous determination of CEA and the corresponding therapeutic drug C225, as shown in Figure 2 [21]. The assay is based on the competition between a quantum dots labeled glycans and target glycans using lectins as the recognition element. The dual-analytic biosensor detected CEA and $\mathrm{C} 225$ in the range from $1 \mathrm{ng} \mathrm{mL}^{-1}$ to $400 \mu \mathrm{g} \mathrm{m}^{-1}$ by stripping voltammetry. The biosensor developed here for the simultaneous detection of the biomarkers and therapeutic drugs is expected to develop as a potential technique for quantitative pharmacology.

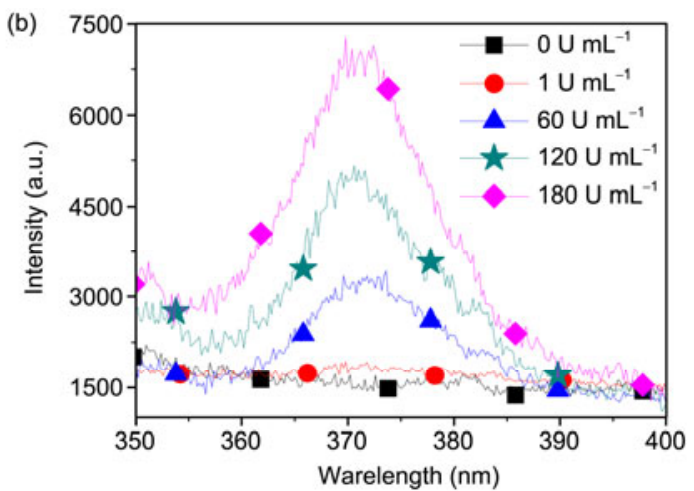

Figure 1 (a) SWV curves of the pending-solution with the series concentration of CA 19-9 inserted with protocol of sandwich-immunosensor; (b) the UV PL spectra of the immunosensors with series CA19-9 concentration. 


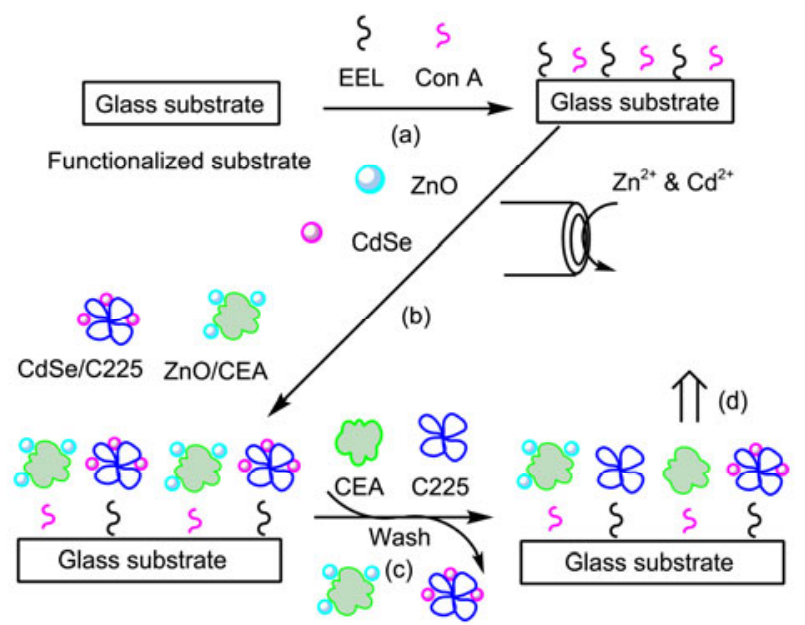

Figure 2 Fabrication and sensing process of $\mathrm{ZnO}$ and $\mathrm{CdS}$ QD-based biosensor for simultaneous detection of CEA and C225. (a) Substrate functionization and lections (EEL and Con A) immobilization; (b) assembly of bioconjugates $(\mathrm{ZnO} / \mathrm{CEA}$ and $\mathrm{CdSe} / \mathrm{C} 225)$ on lectin layer; (c) analytes addition and displacement; (d) SWV detection of the remnant QDs.

\section{1-D nano-ZnO biosensor}

1-D $\mathrm{ZnO}$ nanomaterials were used as carriers with large surface to increase the loading amount of enzymes. 1-D nanostructured $\mathrm{ZnO}$ is favor to immobilization of various enzymes and is facilitate to the electron transfer between the redox centers of enzymes and the electrochemical electrode [22,23].

The conventional $\mathrm{ZnO}$ modified electrode are almost fabricated by post-pasting of $\mathrm{ZnO}$ [24-27], which did not enable robust mechanical adhesion and electrical contact between the nanostructured $\mathrm{ZnO}$ and the electrode. It is desirable to improve the electrical contaction and sensing performance if $\mathrm{ZnO}$ nanostructured electrodes in situ synthesized without using any organic reagent. Therefore, we developed the method that the gold electrode was coated by a thin layer of $\mathrm{Zn}-\mathrm{Au}$ alloy to improve the nucleation for in situ growth of $\mathrm{ZnO}$ nanostructures and to further improve the performance of the biosensor, which was constructed by immobilizing tyrosinase (Tyr) on the $\mathrm{ZnO}$ nanostructures for phenol detection [28]. Electrochemical measurements, Fourier transform infrared and scanning electron microscopic analyses demonstrated that the Tyr was stably adsorbed on the $\mathrm{ZnO}$ nanostructures surface with bioactivity for phenol oxidization. The biosensor reached the sensitivity was as high as $103.08 \mu \mathrm{A} /\left(\mathrm{mmol} \mathrm{L}^{-1}\right)$ at $C_{\text {phenol }}>20 \mu \mathrm{mol} \mathrm{L}^{-1}$ and was $40.76 \mu \mathrm{A} /\left(\mathrm{mmol} \mathrm{L}^{-1}\right)$ at $C_{\text {phenol }}<20 \mu \mathrm{mol} \mathrm{L} \mathrm{L}^{-1}$. The detection limit of $0.623 \mu \mathrm{mol} \mathrm{L}{ }^{-1}$ was obtained.

To improve the performance, the poly (sodium 4-styrenesulfonate) and horseradish peroxidase (HRP) were alternatively immobilized on $\mathrm{ZnO}$ nanostructures repeatedly [29]. The amount of HRP on the $\mathrm{ZnO}$ nanostructures surface increased along with the modified-layer increase. Electrochemical measurement analysis demonstrated that the HRP kept bioactivity for $\mathrm{H}_{2} \mathrm{O}_{2}$ detection without an electron transfer mediator. The multilayered HRP sensors exhibited a wide linear range and low detection limit. The sensitivity of the biosensor increased with the immobilized HRP layers from the lowest value of $58.15 \mu \mathrm{A} /\left(\mathrm{mmol} \mathrm{L}^{-1}\right)$ for five layers.

Unique structured nanocomposite also can facilitate the direct electron transfer between redox proteins and the electrodes [22,23] and therefore improve the performance of the biosensor. Consequently, a $\mathrm{ZnO} / \mathrm{Cu}$ nanocomposite in situ-

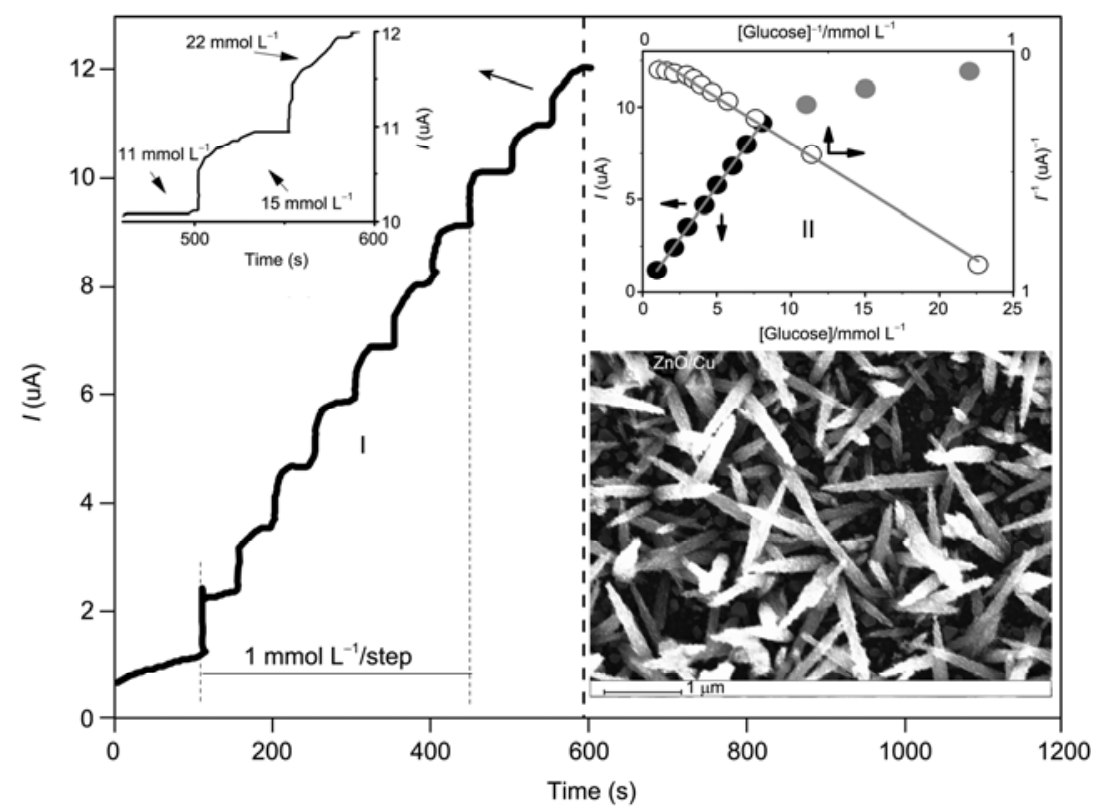

Figure 3 Amperometric response of the biosensor to different concentrations of glucose in PBS (0.1 mol/L, pH 7.4) at an applied potential of -0.39 V. Inset II: The calibration curve (current versus glucose concentration) and Lineweaver-Burk plot (current ${ }^{-1}$ versus concentration $^{-1}$ ) from curve I. Inset upper right: $\mathrm{SEM}$ of the $\mathrm{ZnO} / \mathrm{Cu}$ nanocomposite. 
directed growth on electrode was prepared and applied in biosesnor [30]. SEM images demonstrate that the morphology of $\mathrm{ZnO} / \mathrm{Cu}$ nanocomposite has a large specific surface area, which is favorable to immobilize the biomolecules and realize the direct electron transfer between the electrode surface and the redox protein (Figure 3). As a model, this $\mathrm{ZnO} / \mathrm{Cu}$ nanocomposite is employed for immobilization of GOx and the construction of the glucose biosensor. Direct electron transfer of $\mathrm{GOx}$ is achieved at $\mathrm{ZnO} / \mathrm{Cu}$ nanocomposite with a high heterogeneous electron transfer rate constant of $0.67 \pm 0.06 \mathrm{~s}^{-1}$. The electrochemical assay also showed specificity for the detection of glucose and demonstrated a dynamic range of $1-9 \mathrm{mmol} \mathrm{L}^{-1}$ with detection limit of $0.04 \mathrm{mmol} \mathrm{L}^{-1}$. Such $\mathrm{ZnO} / \mathrm{Cu}$ nanocomposite provides a good matrix for direct electrochemistry of enzymes and mediator-free enzymatic biosensors.

\section{Conclusions}

The unique properties of $\mathrm{ZnO}$ and the ease of $\mathrm{ZnO}$ nanostructure fabrication make this material suitable for biosensor applications. The examples have briefly summarized the interaction/integration of $\mathrm{ZnO}$ QDs and $\mathrm{ZnO}$ 1-D nanostructures with biomolecules. The next challenge of modified electrodes is their applicability for the in vivo detection and its stability. Many researchers concentrate on ease, user-friendly, less interference, and commercially available electrodes. In this aspect, $\mathrm{ZnO}$ nanostructures have an important role to play in near future. The versatility of properties and formation of nanostructures affords the promise for its applications in chemical and biochemical biosensors.

This work was supported by the National Natural Science Foundation of China (60725413 and 60976002), the National Basic Research Program of China (2011CB302004) and the Ministry of Education (309015).

1 Yogeswaran U, Chen S M. A review on the electrochemical sensors and biosensors composed of nanowires as sensing material. Sensors, 2008, 8: 290-313

2 Pearton S J, Norton D P, Ip K, et al. Recent progress in processing and properties of $\mathrm{ZnO}$ Progress in Materials. Prog Mater Sci, 2005, 50: $293-340$

3 Sun X W, Kwok H S. Optical properties of epitaxially grown zinc oxide films on sapphire by pulsed laser deposition. J Appl Phys, 1999, 86: 408-411

4 Tian Z R R, Voigt J A, Liu J, et al. Biominetic arrays of oriented helical $\mathrm{ZnO}$ nanorods and columns. J Am Chem Soc, 2002, 124: 12954-12955

5 Wei A, Sun X W, Wang J X, et al. Enzymatic glucose biosensor based on $\mathrm{ZnO}$ nanorod array grown by hydrothermal decomposition. Appl Phys Lett, 2006, 89: 123902-1-3

6 Dong Z F, Tian Y, Yin X, et al. Physical vapor deposited zinc oxide nanoparticles for direct electron transfer of superoxide dismutase. Electrochem Commun, 2008, 10, 818-820

7 Degen A, Kosec M. Effect of $\mathrm{pH}$ and impurities on the surface charge of zinc oxide in aqueous solution. J Eur Ceram Soc, 2000, 20: 667-673
8 Dorfman A, Kumar N, Hahm J. Nanoscale ZnO-enhanced fluorescence detection of protein interactions. Adv Mater, 2006, 18: 26852690

9 Asif M H, Nur O, Willander M, et al. Selective calcium ion detection with functionalized $\mathrm{ZnO}$ nanorods-extended gate MOSFET. Biosens Bioelectron, 2009, 24: 3379-3382

10 Dai Z, Shao G, Hong J, et al. Immobilization and direct electrochemistry of glucose oxidase on a tetragonal pyramid-shaped porous $\mathrm{ZnO}$ nanostructure for a glucose biosensor. Biosens Bioelectron, 2009, 24: 1286-1291

11 Rahman M M, Umar A, Sawada K. Ultra-sensitive cholesterol biosensor based on low-temperature grown $\mathrm{ZnO}$ nanoparticles. Electrochem Commun, 2009, 11: 118-121

12 Zhao J, Wu D, Zhi J. A novel tyrosinase biosensor based on biofunctional $\mathrm{ZnO}$ nanorod microarrays on the nanocrystalline diamond electrode for detection of phenolic compounds, Bioelectrochemistry, 2009, 75: 44-49

13 Somers R C, Bawendi M G, Nocera D G. CdSe nanocrystal based chem-/bio-sensors. Chem Soc Rev, 2007, 36: 579-591

14 Gill R, Zayats M, Willner I. Semiconductor quantum dots for bioanalysis. Angew Chem Int Ed, 2008, 47: 7602-7625

15 Barbara S, Antonina H S, Witold K, et al. Tissue polypeptide specific antigen (TPS), a marker for differentiation between pancreatic carcinoma and chronic pancreatitis: A comparative study with CA 19-9. Cancer, 2000, 89: 83-88

16 Gu B X, Xu C X, Yang C, et al. ZnO quantum dot labeled immunosensor for carbohydrate antigen 19-9. Biosens Bioelectron, 2011, 26: 2720-2723

17 Zhang X, Teng Y, Fu Y, et al. Lectin-based biosensor strategy for electrochemical assay of glycan expression on living cancer cells. Anal Chem, 2010, 82: 9455-9460

18 Yang C, Gu B X, Xu C X, et al. Self-assembled ZnO quantum dot bioconjugates for direct electrochemical determination of allergen. $\mathrm{J}$ Electroanal Chem, 2011, 660: 97-100

19 Yang C, Xu C X, Wang X M, et al. A displacement assay for the sensing of carbohydrate using zinc oxide biotracers. Electrochim Acta, 2012, 60: 50-54

20 Chen J P. Performance of affinity biosensors with. competitive displacement mechanism. J Chem Tech Biotechnol, 1993, 56: 213-211

21 Yang C, Xu C X, Wang X M, et al. Quantum-dot-based biosensor for simultaneous detection of biomarker and therapeutic drug: First steps toward an assay for quantitative pharmacology. Analyst, 2012, 137: 1205-1209

22 Yang M, Qu F, Li Y, et al. Direct electrochemistry of hemoglobin in gold nanowire array. Biosens Bioelectron, 2007, 23: 414-420

23 Xiao Y, Li C M. Nanocomposites: From fabrications to electrochemical bioapplications. Electroanalysis, 2008, 20: 648-662

$24 \mathrm{Lu} \mathrm{X}$, Zhang H, Ni Y, et al. Porous nanosheet-based ZnO microspheres for the construction of direct electrochemical biosensors. Biosens Bioelectron, 2008, 24: 93-98

25 Wu S, Ju H X, Liu Y. Conductive mesocellular silica-carbon nanocomposite foams for immobilization, direct electrochemistry, and biosensing of proteins. Adv Funct Mater, 2007, 17: 585-592

26 Bao S J, Li C M, Zang J F, et al. New nanostructured $\mathrm{TiO}_{2}$ for direct electrochemistry and glucose sensor applications. Adv Funct Mater, 2008, 18: 591-599

27 Chen L Y, Gu B X, Zhu G P, et al. Tyrosinase biosensor based on zinc oxide nanorods. Nano, 2007, 2: 281-284

28 Gu B X, Xu C X, Zhu G P, et al. Tyrosinase immobilization on zno nanorods for phenol detection. J Phys Chem B, 2009, 113: 377-381

29 Gu B X, Xu C X, Zhu G P, et al. Layer by layer immobilized horseradish peroxidase on zinc oxide nanorods for biosensing. J Phys Chem B, 2009,113: 6553-6557

30 Yang C, Xu C X, Wang X M. ZnO/Cu Nanocomposite: A platform for direct electrochemistry of enzymes and biosensing applications. Langmuir, 2012, 28: 4580-4585

Open Access This article is distributed under the terms of the Creative Commons Attribution License which permits any use, distribution, and reproduction in any medium, provided the original author(s) and source are credited. 